\title{
Rotation of stripe domains in a sputter deposited Tb-Fe-Ga thin film
}

1

2 D. Bisero ${ }^{1,2}$, S. Fin ${ }^{1}$ and R. Ranchal ${ }^{3}$

3

4

5 Universitaria s/n Madrid 28040, Spain perpendicular magnetic anisotropy; magnetic devices.

${ }^{1}$ Dipartimento di Fisica, Università di Ferrara, Via Saragat 1, 44122 Ferrara, Italy

${ }^{2}$ CNISM ,Unità di Ferrara, Via Saragat 1, 44122 Ferrara, Italy

${ }^{3}$ Dpto. Física de Materiales, Facultad Ciencias Físicas. Universidad Complutense de Madrid. Ciudad

\begin{abstract}
The interest in Tb-Fe-Ga alloys, prepared by techniques compatible with technological processes, has been increasing in last years, due to their high magnetostriction combined with their potential application in spintronic devices. In this letter, we report the domain configurations of a $\mathrm{Tb}_{10} \mathrm{Fe}_{76} \mathrm{Ga}_{14}$ thin film grown by co-sputtering deposition. The film exhibits a weak perpendicular magnetic anisotropy (PMA) and the presence of discontinuous stripe domains. The application of an external field in the film plane, perpendicular to the stripe direction, induces the rotation of the stripes and the alignment of their axis with the field. This property of the material has been observed in the range of applied fields 0-70 $\mathrm{mT}$, above which PMA disappears and stripe annihilation occurs.
\end{abstract}

Keywords: magnetic thin films; domain structure; stripe and bubble domains; rotation of stripe domains; 
1 Corresponding author:

2 Dr. D. Bisero

3 Dipartimento di Fisica

4 Università di Ferrara

$5 \quad$ Via Saragat 1

644122 Ferrara

$7 \quad$ Italy

8 bisero@fe.infn.it

9 Phone: 0039532974313

10

11

12 
The solutions of the micromagnetic equations for a low-anisotropy thin film with perpendicular anisotropy have the form of stripe domains. They were first discovered theoretically $[1,2]$ and then observed experimentally $[3,4]$ in the 1960 s.

More recently, it has been shown that the domain boundaries between two adjacent stripe domains can act as geometrical limits, in analogy with an artificially patterned array of nanowires, limiting the spin wave propagation at the domain wall and producing spin wave quantization $[5,6,7]$. This effect could be used to control the spin-wave frequency in spintronic devices, like filters or oscillators, acting first of all on the

7 thickness of a continuous thin film, which regulates the stripe (and consequently the domain boundaries) dimensions. An interesting feature, which can concern thin films displaying stripe domains, particularly those with high magnetostriction constant and low in-plane magnetic anisotropy, is the possibility of rotating the stripe direction with an in-plane magnetic field, which is high enough to saturate the magnetization $[3,8]$. After field removal, the stripe axis and the in-plane remanent magnetization maintain the original direction induced by the saturating field. In view of spintronic applications, this could provide a way to control the propagation direction of spin waves.

Stripe domains and their rotation have been recently observed and studied in epitaxially grown thin films $[9,10,11,12]$ displaying very high crystalline quality, obtained by advanced deposition methods, which are difficult to implement in a device-production process. The search for new materials displaying these features, produced by techniques compatible with industrial and technological processes, could be a key point and a further step towards the possibility of modulating the spin wave frequencies and their propagation direction, through the control of the periodicity and the rotation of stripes in thin film magnetic devices, without using the technologically complex patterning process $[13,14]$. 
1 The structural and magnetic properties of Tb-Fe-Ga films have been studied in last years by this collaboration $[15,16,17]$. In this paper we demonstrate for the first time, to the best of our knowledge, the rotation of stripe domains in a TbFeGa thin film produced by sputter deposition, that is a technique compatible with industrial and technological processes.

\section{Material and methods} (1)

Two targets with a nominal composition of $\mathrm{TbFe}_{2}$ and $\mathrm{Fe}_{3} \mathrm{Ga}$ were employed to deposit by co-sputtering the $\mathrm{Tb}_{10} \mathrm{Fe}_{76} \mathrm{Ga}_{14}$ alloy. The co-sputtering process was performed in the oblique incidence, being the angle between each target and the substrate of about 25 degrees $[15,16,17]$ and the distance between the targets and the substrate of $15 \mathrm{~cm}$. A DC power of $100 \mathrm{~W}$ was used in the $\mathrm{Fe}_{3} \mathrm{Ga}$ target whereas in the $\mathrm{TbFe}_{2}$ target $100 \mathrm{~W}$ were applied in a pulsed source, working at a frequency of $25 \mathrm{kHz}$, with a dead-time of $5 \mu \mathrm{s}$. The $250 \mathrm{~nm}$-thick layer was deposited at room temperature on a $5 \times 4 \mathrm{~mm}^{2}$ glass substrate. Mo layers (20 $\mathrm{nm}$ ) were used as buffer and capping of the Tb-Fe-Ga film, being deposited with a DC power of $90 \mathrm{~W}$. The Ar pressure was 0.2 Pa to evaporate all the layers.

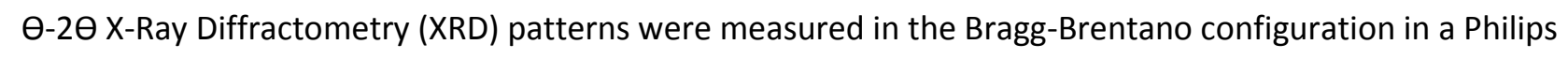
X'Pert MPD using the Cu $\mathrm{K}_{\alpha}$ wavelength (1.54056 ̊̊). At room temperature, in-plane and out-of-plane hysteresis loops were carried out in a vibrating sample magnetometer (VSM) from LakeShore. We measured in-plane hysteresis loops by VSM at different angles between the in-plane reference direction (the long side of the sample) and the in-plane applied magnetic field. In-field Magnetic Force Microscopy (MFM) measurements, at $50 \mathrm{~nm}$ lift scan height, were performed without removing the sample from the magnetic field region, in order to analyze the changes in the domain structure of a specific area, caused by intensity field variations, using a technique and instrumentation described in references $[18,19,20]$. During the MFM measurements we observed negligible drift effects, carefully monitored by AFM and consequently compensated by small displacements of the scanned area, in order to be sure of exploring the same area at every field. 
Magnetovectometry measurements were obtained by means of a home-built Surface Magneto-Optical Kerr Effect (S-MOKE) apparatus. The principle of operation has been exhaustively described in reference $[21,22]$.

\section{Results and discussion}

The VSM results reported in Fig. 1 point out the presence of a weak Perpendicular Magnetic Anisotropy (PMA) in the $\mathrm{Tb}_{10} \mathrm{Fe}_{76} \mathrm{Ga}_{14}$ film. A magnetic material will have PMA when is isotropic in the sample plane and the out-of-plane direction is an easy axis compared to any in-plane direction [17]. In our case, loops recorded in the out of plane and in any in-plane direction are almost the same within the resolution of the VSM. The fact that the VSM signal for moderate perpendicular fields is slightly lower than for in-plane ones allows us to say that this sample shows a weak PMA, as furtherly confirmed by MFM measurements. XRD measurements show a microstructure close to the $\mathrm{TbFe}_{2}$, as already reported in previous works (Fig. 1, inset) $[16,17]$. The low intensity of the diffraction peak reflects the low crystallinity of this sample that is also confirmed by the high full width at half maximum (FWHM) of around $1^{\circ}$. We have calculated the lattice parameter considering the layer has the cubic structure of the $\mathrm{TbFe}_{2}$, obtaining a value of $7.39 \AA$, that is a larger value in comparison to the theoretical one for $\mathrm{TbFe}_{2}(7.34 \AA)$. This indicates that the structure is distorted. It is well known that a thin ferromagnetic film displaying PMA can experience the competition between the PMA energy, which fosters local alignment along the out-of-plane direction, and long-range magnetostatic energy, which favors in-plane magnetization, originating magnetic stripe domains [23]. We studied in depth the domain configuration of the film by MFM (Figures 2 and 3), both in presence of an external magnetic field and at remanence. authors as a maze pattern [11] and by other authors as a mixed pattern [24], since it contains both short 
stripes and bubble domains. Indeed it can be considered as an irregular stripe domain pattern, with no preferential direction of the stripes, mixed with bubble domains.

3 This is a metastable state and its presence is a characteristic feature of the domain structures found in films with PMA. It disappears when an in-plane external field of $80 \mathrm{mT}$ is applied (Fig. 2b), but as soon as the field's intensity is reduced to $40 \mathrm{mT}$ a new metastable state (Fig. 2c) made of irregular stripes (characteristic width $220 \pm 10 \mathrm{~nm}$, determined by 2D-FFT, preferentially oriented along the field, mixed with some residual bubble) appears. This state corresponds to a local minimum of the energy of the system and persists at remanence (Fig. 2d), where the maze pattern stretches into discontinuous stripes in the field direction. It is

9 known that the process leading to this final mixed pattern [24] is characterized by a cascade of discontinuous coalescence steps. The detailed magnetic configuration of the intermediate and the final states depends on additional, secondary anisotropy contributions [25], which are superimposed onto the fundamental perpendicular anisotropy of these films. Transitions between a bubble lattice and a stripe domain pattern are possible if the respective lattices are not perfect but contain domain dislocations. These defects in the magnetic pattern allow to adjust the stripe period towards the equilibrium value. Bubbles are a natural source for such dislocations and the role of defects is generally acknowledged as a key feature in the behavior of this kind of materials. If we start from the remanence state of Figure $2 \mathrm{~d}$ and apply an in-plane external field perpendicular to the previous one performing in-field MFM measurements (from Fig. 3a to 3d), we observe a substantial rotation of the elongated domains starting at about $30 \mathrm{mT}$. The preferential domain orientation at $47 \mathrm{mT}$ (Fig. 3d) is found rotated with respect to the horizontal initial one, toward the field direction, and the remanence state doesn't substantially change (Fig. 3e). By the way, the stripe rotation process is not coherent and at an intermediate stage local orientation disorder is present, as already shown in ref. [9]. It is necessary to reach a field of $80 \mathrm{mT}$ (see fig. 2) in order to completely align the stripes along the external field. We could say that we observed a transition from a chaotic (disordered) phase (Fig. 2a) to a more ordered one (Fig. 3e), both observed at remanence, and that the domain orientation of the system does depend on the history of the sample, that is on the direction of a previously applied magnetic field. This 
particular feature may give rise to significant applications. For example, it can be used to build a method for the determination of frauds produced by magnetic fields in specific industrial devices [26].

3 Fine and irregular stripe domain structures, comparable to those shown in this paper, have been observed by many other authors (see for example refs. $[11,27,28]$ ). These magnetic structures partially differ from other domain configurations typical of similar physical systems, which instead display nearly continuous stripe domains and a linear reversible part of the magnetization loop in correspondence of the fields at which the stripe rotation is observed $[9,29,30]$. This linear part of the magnetization loop has been considered as a signature of the presence of magnetic rotatable anisotropy in the latter systems.

9 Conversely, this work and other papers reporting discontinuous or irregular stripe domains, do not show the linear and reversible part of the magnetization loop as a clear evidence. By the way, the full in-plane magnetic isotropy of our Tb-Fe-Ga film (Fig. 4) and the evident observation of the domain rotation process allow to establish an analogy with the rotatable anisotropy mechanism described in the case of continuous stripes. The main difference between the two systems is that irreversible changes of the in-plane component of the magnetization occur in the case of discontinuous stripes mainly by local motion of short sections of domain walls [27]. This reversal process is different from that of continuous stripes, which are characterized by the simultaneous motion of long domain wall sections and the growth of domains of the preferred magnetization direction at the expense of other ones. The existence of stripe domains can be ascribed to the competition between the out-of-plane anisotropy and the long-range dipolar interaction, which act on different length scales. The spins are mainly oriented out of the film plane and their direction switches periodically from up to down, thus forming a stripe-like domain pattern. The modulation wavelength of the pattern is due to the different scales of interactions, while the stripe direction is a result of the spontaneous breaking of the rotational symmetry. The equivalence of the in-plane directions gives rise to the formation of stripes and the experimental observation of the domains rotation. This phase is characterized by the appearance of many metastable 
1 field allows the system to take many different metastable configurations, very close to each other in

2 energy, producing the rotation of the domains.

3 The appearance of the ordered stripe domain phase at low fields can be related to the increase of the out

4 of plane component of the magnetization with decreasing the in-plane external field, which changes the

5 equilibrium among the different competing magnetic interactions and relative anisotropies. In order to

6 follow this process and determine the transition field at which the magnetization falls fully in-plane, we

7 studied the polar component of the magnetization, that is the value of the out of plane magnetization of

8 the film when an in-plane field is swept between opposite saturation values. This was obtained by means of

9 a home-built Surface Magneto-Optical Kerr Effect Magnetometer (S-MOKE) by which we can determine how the three components of the magnetization vector change along the hysteresis cycle.

A He-Ne laser beam $(1.96 \mathrm{eV})$ is linearly polarized at a given angle with respect to the optical plane (" $p$ " in the plane, "s" normal to the plane), then is reflected by the sample surface at an angle of about 30 degrees. Due to the Kerr Effect, the linearly polarized light becomes elliptical. A Photoelastic Modulator gives to the two components of the reflected beam a cosine phase displacement. In this way, the signal can be separated in a term proportional to the rotation and in another one proportional to the ellipticity of the reflected beam. It is possible to demonstrate (using the Jones formalism) that summing the ellipticity (or equivalently the rotation) of the reflected p-polarized beam to the ellipticity (rotation) of the reflected spolarized beam, the result is proportional to the polar component of the magnetization $\left(m_{z}\right)$. We see in figure 5 that above an in-plane field of about $70 \mathrm{mT}$ the component $\mathrm{m}_{\mathrm{z}}$ reduces to zero, whereas, when the external field gradually goes to lower values, the absolute value of the measured polar magnetization increases and reaches a maximum at remanence.

This is in agreement with the MFM results shown in figures 2 and 3, from which we infer that stripes are still present at $47 \mathrm{mT}$, but completely disappear at $80 \mathrm{mT}$. 


\section{Conclusions}

5

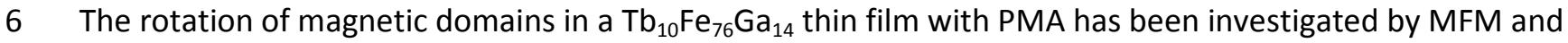
MOKE. The competition between the PMA, demonstrated by VSM measurements, and the long-range magnetostatic energy has generated discontinuous stripe domains. The film has shown full in-plane magnetic isotropy.

With the application of an external magnetic field between 30 and $80 \mathrm{mT}$ in the plane of the film we observed the rotation of the stripes. The out of plane magnetization disappeared for in-plane fields above $70 \mathrm{mT}$. properties of Tb-Fe-Ga alloys.

\section{Acknowledgements}

The authors thank M.G. Pini for valuable discussions. This work has been financially supported through the projects FIRB2010 RBFR10E61T 'NANOREST' of the Italian Ministry of Education, University and Research and MAT2015-66888-C3-3-R of the Spanish Ministry of Economy and Competitiveness (MINECO). 


\section{Figure captions}

2

3

4

Fig. 1

Comparison of in-plane and out-of-plane hysteresis loops recorded at room temperature by VSM. Inset: Xray diffraction pattern of the $\mathrm{Tb}_{10} \mathrm{Fe}_{76} \mathrm{Ga}_{14}$ layer. The error bar of the measurements can be taken as the size of the experimental points in the figure.

Fig. 2

MFM measurements $\left(5.6 \times 5.6 \mu \mathrm{m}^{2}\right)$ of the stripe induction process: a) after deposition; b) in-field at $\mathrm{H}=80$ $\mathrm{mT}$; c) in-field at $\mathrm{H}=40 \mathrm{mT}$; d) at remanence. Images from b) to d) have been recorded sequentially in the same experiment, following half of a minor hysteresis loop. The characteristic domain size determined by $2 \mathrm{D}-\mathrm{FFT}$ is $220 \pm 10 \mathrm{~nm}$ (except for the as-deposited sample, where a value of $270 \mathrm{~nm}$ has been found).

\section{Fig. 3}

In-field MFM measurements $\left(5.6 \times 5.6 \mu \mathrm{m}^{2}\right)$ of the stripe rotation process: a) $\left.\mathrm{H}=11 \mathrm{mT} ; \mathrm{b}\right) \mathrm{H}=25 \mathrm{mT}$; c) H = $33 \mathrm{mT}$; d) $\mathrm{H}=47 \mathrm{mT}$; e) at remanence. All the images have been recorded sequentially during the same experiment, with the external field applied perpendicularly to the stripe axis. The characteristic domain size determined by $2 \mathrm{D}-\mathrm{FFT}$ is $220 \pm 10 \mathrm{~nm}$.

\section{Fig. 4}

Comparison at room temperature of VSM loops recorded with different orientation of the in-plane magnetic field $(0,30,60$ degrees with respect to a reference in-plane direction). The error bar of the measurements can be taken as the size of the experimental points in the figure. 
$1 \quad$ Fig. 5

2 MOKE magnetovectometry measurements of the stripe annihilation process: out of plane magnetization of

3 the film, when an in-plane field is swept between opposite values.

4

5 


\footnotetext{
${ }^{1}$ M.W. Muller, Distribution of the Magnetization in a Ferromagnet, Phys. Rev. 122 (1961) 1485-1489.

${ }^{2}$ W.F. Brown, Rigorous Calculation of the Nucleation Field in a Ferromagnetic Film or Plate, Phys. Rev. 124 (1961) 1348-1353.

${ }^{3}$ R.J. Spain, Dense-banded domain structure in "rotatable anisotropy" permalloy films, Appl. Phys. Lett. 3 (1963) 208209.

${ }^{4}$ N. Saito, H. Fujiwara, Y. Sugita, A New Type of Magnetic Domain in Thin Ni-Fe Films, J. Phys. Soc. Japan 19 (1964) 421-422.

${ }^{5}$ Seung-Seok Ha, Jungbum Yoon, Sukmock Lee, Chun-Yeol You, Myung-Hwa Jung and Young Keun Kim, Spin wave quantization in continuous film with stripe domains, J. Appl. Phys. 105 (2009) 07D544.

${ }^{6}$ P. E. Roy, T. Trypiniotis, C. H. W. Barnes, Micromagnetic simulations of spin-wave normal modes and the resonant field-driven magnetization dynamics of a $360^{\circ}$ domain wall in a soft magnetic stripe, Phys. Rev. B 82 (2010) 134411

${ }^{7}$ J. Yoon, C.-Y. You, Y. Jo, S.-Y. Park, M.-H. Jung, Current-induced localized spin wave interference in a ferromagnetic nanowire with a domain wall, J. Magn. Magn. Mater. 325 (2013) 52-56.

${ }^{8}$ R. J. Prosen, J. O. Holmen, and B. E. Gran, Rotatable Anisotropy in Thin Permalloy Films, J. Appl. Phys. 32 (1961) $91 \mathrm{~S}$.

${ }^{9}$ S. Tacchi, S. Fin, G. Carlotti, G. Gubbiotti, M. Madami, M. Barturen, M. Marangolo, M. Eddrief, D. Bisero, A. Rettori, Rotatable magnetic anisotropy in a $\mathrm{Fe}_{0.8} \mathrm{Ga}_{0.2}$ thin film with stripe domains: Dynamics versus statics, Phys. Rev. B 89 (2014) 024411.

${ }^{10}$ S. Fin, R. Tomasello, D. Bisero, M. Marangolo, M. Sacchi, H. Popescu, M. Eddrief, C. Hepburn, G. Finocchio, M. Carpentieri, A. Rettori, M.G. Pini, S. Tacchi, In-plane rotation of magnetic stripe domains in $\mathrm{Fe}_{1-\mathrm{x}} \mathrm{Ga}_{\mathrm{x}}$ thin films, Phys. Rev. B 92 (2015) 224411.

${ }^{11}$ M. A. Marioni, N. Pilet, T.V. Ashworth, R. C. O'Handley, and H. J. Hug, Remanence due to Wall Magnetization and Counterintuitive Magnetometry Data in 200-nm Films of Ni, Phys. Rev. Lett. 97 (2006) 027201.

${ }^{12}$ U. Ebels, L. Buda, K. Ounadjela, P. E. Wigen, Ferromagnetic resonance excitation of two-dimensional wall structures in magnetic stripe domains, Phys. Rev. B 63 (2001) 174437.

${ }^{13}$ D. Bisero, P. Cremon, M. Madami, S. Tacchi, G. Gubbiotti, G. Carlotti and A.O. Adeyeye, Magnetization reversal of rectangular particles: closure states and effect of dipolar coupling, IEEE Trans. Magn. 48 (2012) 1593-1596

${ }^{14}$ M. Sepioni, M. Madami, S. Tacchi, G. Gubbiotti, G. Carlotti, D. Bisero, A.O. Adeyeye, N. Singh, S. Goolaup, Dipolar interaction in dense chains of submicrometric rectangular dots, J. Phys.: Conf. Ser. 200 (2010) 072089

${ }^{15}$ R. Ranchal, S. Fin, D. Bisero, C. Aroca, Tailoring the magnetic anisotropy and domain patterns of sputtered TbFeGa alloys, J. Alloys Compd. 582 (2014) 839-843.

${ }^{16}$ R. Ranchal, S. Fin, D. Bisero, Evolution of the structural and magnetic properties of sputtered $\mathrm{Tb}_{\mathrm{x}} \mathrm{Fe}_{73} \mathrm{Ga}_{27-x}(7 \mathrm{at} . \%<$ $x<11$ at.\%) thin films upon the increase of Tb content, J. Alloys Compd. 667 (2016) 262-267.

${ }^{17}$ R. Ranchal and V. Gutiérrez-Díez, Perpendicular magnetic anisotropy in TbFeGa ternary alloys grown by cosputtering, Thin Solid Films 534 (2013) 557-560.

${ }^{18}$ G. Gubbiotti, P. Malagò, S. Fin, S. Tacchi, L. Giovannini, D. Bisero, M. Madami, G. Carlotti, J. Ding, A. O. Adeyeye, R. Zivieri, Magnetic normal modes of bicomponent permalloy/cobalt structures in the parallel and antiparallel ground state, Phys. Rev. B 90 (2014) 024419.

${ }^{19}$ M. Madami, D. Bisero, G. Gubbiotti, S. Tacchi, G. Carlotti, K. Nakano and T. Ono, Magnetization configurations in $\mathrm{NiFe}$ slotted rings studied by magneto-optical Kerr effect and Magnetic Force Microscopy, IEEE Trans. Magn. 48 (2012) 1269-1272.

${ }^{20}$ D. Bisero, P. Cremon, M. Madami, M. Sepioni, S. Tacchi, G. Gubbiotti, G. Carlotti, A.O. Adeyeye, Effect of dipolar interaction on the magnetization state of chains of rectangular particles located either head-to-tail or side-by-side, J. Nanopart. Res. 13 (2011) 5691-5698.

${ }^{21}$ P. Vavassori, Polarization modulation technique for magneto-optical quantitative vector magnetometry, Appl. Phys. Lett. 77 (2000) 1605-1607.

${ }^{22}$ V. Bonanni, D. Bisero, P.Vavassori, G. Gubbiotti, M. Madami, A.O. Adeyeye, S. Goolaup, N. Singh, T. Ono, C. Spezzani, Shape and thickness effects on the magnetization reversal of $\mathrm{Py} / \mathrm{Cu} / \mathrm{Co}$ nanostructures, J. Magn. Magn. Mater. 321 (2009) 3038-3041.

${ }^{23}$ T. Garel and S. Doniach, Phase transitions with spontaneous modulation - the dipolar Ising ferromagnet, Phys. Rev. B 26 (1982) 325-329.

${ }^{24}$ A. Hubert and R. Schaefer, Magnetic Domains, Springer, Berlin, 1998.

${ }^{25}$ A. Hubert, A.P. Malozemoff, J. De Luca, Effects of cubic, tilted uniaxial, and orthorhombic anisotropies on homogeneous nucleation in a garnet bubble film, J. Appl. Phys. 45 (1974) 3562-3571.

${ }^{26}$ R. Ranchal, S. Fin and D. Bisero, Spanish Patent Application, P201500492.
} 
${ }^{27}$ C. C. H. Lo, J. E. Snyder, J. Leib, R. Chen, B. Kriegermeier-Sutton, M. J. Kramer, D. C. Jiles, M. T. Kief, Magnetic force microscopy study of magnetization reversal in sputtered FeSiAl(N) films, J. Appl. Phys. 89 (2001) 2868-2872.

${ }^{28}$ R. Bručas, H. Hafermann, M. I. Katsnelson, I. L. Soroka, O. Eriksson, and B. Hjörvarsson, Magnetization and domain structure of bcc $\mathrm{Fe}_{81} \mathrm{Ni}_{19} / \mathrm{Co}$ (001) superlattices, Phys. Rev. B 69 (2004) 064411.

${ }^{29}$ M. Barturen, B. Rache Salles, P. Schio, J. Milano, A. Butera, S. Bustingorry, C. Ramos, A. J. A. de Oliveira, M. Eddrief, E. Lacaze, F. Gendron, V. H. Etgens, and M. Marangolo, Crossover to striped magnetic domains in $\mathrm{Fe}_{1-x} \mathrm{Ga}_{x}$ magnetostrictive thin films, Appl. Phys. Lett. 101 (2012) 092404.

${ }^{30}$ E. Sallica Leva, R. C. Valente, F. Martínez Tabares, M. Vásquez Mansilla, S. Roshdestwensky, A. Butera, Magnetic domain crossover in FePt thin films, Phys. Rev. B 82 (2010) 144410. 